\title{
Telethermographic study of skin circulation in smokers
}

By A. Di Carlo*

*S.Gallicano Dermatological Institute IRCCS-IFO, via Elio Chianesi 53 00144, Rome, Italy,

\begin{abstract}
Skin vascular diseases are accompanied by changes of the temperature of the skin. However, modifications of thermal gradients are often $<0,1^{\circ} \mathrm{C}$. The thermostimulation method let us to overcome the accuracy of the current devices. Infact, performing by contact a cold/hot stimulus under controlled conditions, it is possible to evaluate thermal gradients of a hot/cold spot, by timering its thermal recovery. On these bases, we have performed a study of early effects of cigarette smoking. Infact, among the acute effects of cigarette smoking there is a capillary vasoconstriction. However this effect is not similar in all the subjects.
\end{abstract}

\section{Introduction}

Tobacco smoking is one of the strongest risk factors for the development of cardiovascular morbidity and mortality (1). It has been shown that in the United States more than $30 \%$ of all coronary heart disease deaths are attributable to cigarette smoking, with the risk being strongly dose-related (2). Smoking nearly doubles the risk for ischemic stroke, peripheral vascular disease, and is associated with signs of inflammation, increased intima-media thickness and formation of atherosclerotic plaques in the carotid and femoral arteries (3). Furthermore, smoking aggravates the cardiovascular risk in patients with hypertension and hyperlipidemia and also modulates the effects of other factors, such as high density lipoprotein (HDL)-cholesterol (4), low density lipoprotein (LDL) (5), and insulin resistance (4). Several studies have demonstrated that baseline blood flow in the skin of smokers is reduced either permanently and/or after smoking one or more cigarettes. Reactive hyperemia, studied by means of $\mathrm{pTPO}_{2}$, is compromised in the skin of neonatal infants born to smoking mothers (6), while in adults measuring finger blood flow via plethysmography an average of $55 \%$ decrease after smoking and a reduction of ophthalmic artery circulation in response to cigarette smoking (7)). Other Authirs suggested that decreased retinal blood flow after cigarette smoking is due to the vasoconstrictive effects of nicotine (8) .

Because of the inaccessibility to noninvasive technology, the effect of cigarette smoking on the microcirculation in other organs has been studied predominantly in experimental animals (9). In smoke-exposed animals, capillaries both in the central areas and the periphery of lung lobules were narrowed and the overall capillary density of the lungs significantly reduced. With increased interest in predictive medicine, development of a relatively noninvasive technique that can improve prediction of major clinical outcomes has gained considerable attention.

The skin may prove to be an ideal site for evaluation of microvascular dysfunction due to its ease of access and growing evidence that changes in skin vascular reactivity may precede overt clinical signs of disease. Evaluation of the skin blood flow response to locally applied thermal stress can be used to predict cutaneous but also cardiovascular morbidity.

Telethermography (TTG) is an elective method in the evaluating the microcirculation of the skin. Infact, the infrared emission from the human body strictly depends on skin blood flow that is sited at papillar level of the dermis. Unfortunately, the thermal gradients variations in the skin vessels, as happens in the case of smoking, are often of the order of less than $0,01^{\circ} \mathrm{C}$. Previous study has demonstrated that the new technique of thermal stimulation let us to improve the sensitivity of TTG in this particular field (10-11)

\section{Method}

The material consisted of one group of current smokers $(n=20)$ and a control group, of matched age and matched genders, of 20 nonsmokers. The thermographic tests has been performed the patients staying in a proper room for 2 hrs, smoking consecutively 4 cigarettes, one every 14 minutes. The tests will made at 0 time (baseline), after smoking each cigarette and, finally, 60' after the beginning of the last one.

We employed a telethermography FLYR assisted by a thermal stimulator Surgitron. The parameters of thermal stress were: $+5^{\circ} \mathrm{C} \times 20$ "

\section{Results}

A very evident modification of thermal recovery time in smokers was seen (17/20), with prolonged times of thermal recovery after the cold stimulation in the confront of no smokers subjects $(2 / 20)$, in which moreover the overshoot postischemic effect was constantely seen.

Furthermore, the finding of normal times of thermal recovery in 3/20 smokers could lead to the hypothesis that in these subjects it could be an absence or a reduced response e.g. immunological, of the endothelial nicotine chemoreceptors (12).

Finally, the TTG assisted by thermal stimulation method demonstrates to be a very sensitive technique to visually evaluate effect of smoking on the skin blood flow, visually evident on the monitor as a "thermal amputation" of the hand 
fingers. The method is absolutely non invasive, reproducible and storable, and could validy support the social campaign against the smoke, in particular among the adolescents, to the aim to prevent cardiovascular events.

\section{References}

1) Hawkins BT, Brown RC, Davis TP. Smoking and ischemic stroke: a role for nicotine? Trends Pharmacol Sci. 2002;23:78-82

2) Ockene IS, Miller NH. Cigarette smoking, cardiovascular disease, and stroke: a statement for healthcare professionals from the American Heart Association. American Heart Association Task Force on Risk Reduction.Circulation. 1997;96:3243-7.

3) Bolinder G, Norén A, de Faire U, Wahren J. Smokeless tobacco use and atherosclerosis: an ultrasonographic investigation of carotid intima media thickness in healthy middle-aged men. Atherosclerosis. 1997;132:95-103

4) Tonstad S, Svendsen M. Premature coronary heart disease, cigarette smoking, and the metabolic syndrome. Am J Cardiol. 2005 Dec 15;96:1681-5.

5) Xu, C. B., Stavenow, L., \& Pessah-Rasmussen, H. Interactions between cultured bovine arterial endothelial and smooth muscle cells; effects of modulated low density lipoproteins on cell proliferation and prostacyclin release. Scand J Clin Lab Invest 1994; 54, 191-198.

6) Ahlsten G, Ewald U, Tuvemo T.Maternal smoking reduces prostacyclin formation in human umbilical arteries. A study on strictly selected pregnancies. Acta Obstet Gynecol Scand. 1986;65:645-9.

7) Acevedo A, Schnell A. Effect of cigarette smoking upon the finger circulation in normal and diabetic subjects. Basic Res Cardiol. 1975;70:350-3.

8).Morgado PB, Chen HC, Patel V, Herbert L, Kohner EM. The acute effect of smoking on retinal blood flow in subjects with and without diabetes. Ophthalmology. 1994;101:1220-6.

9) Nicoll PA. The action of inhaled cigarette smoke on the microcirculation, heart rate and carotid pressure of the bat. Angiology. 1966;17:851-8.

10 - Ippolito F, Di Carlo A. La metodica della criostimolazione nell'indagine termografica in dermatologia. Boll. Ist. Dermatol. S. Gall.1980; X: 165-183

11) Di Carlo A. Thermography and the Possibilities for Its Applications in Clinical and Experimental Dermatology. Clinics in Dermatology 1995; 13:329-336

12) Lesage MG, Shelley D, Pravetoni M, Pentel PR. Enhanced attenuation of nicotine discrimination in rats by combining nicotine-specific antibodies with a nicotinic receptor antagonist. Pharmacol Biochem Behav. 2012 Apr 4. [ 